\title{
Análise do índice BODE em pacientes com DPOC severa submetidos a um programa de reabilitação pulmonar
}

\section{Analysis of BODE index in patients with COPD severe submitted to the Pulmonary Rehabilitation Program}

Juliana Roberto Gomes da Silva ${ }^{1}$, Thiago Brasileiro de Vasconcelos ${ }^{2}$, Raimunda Hermelinda Maia Macena ${ }^{2}$, Vasco Pinheiro Diógenes Bastos ${ }^{1}$, Maria Tereza Aguiar Pessoa Morano ${ }^{3}$, Emília Maria Matos Rocha ${ }^{1}$, Maria do Socorro Quintino Farias ${ }^{1}$.

1. Centro Universitário Estácio do Ceará, Brasil. 2. Universidade Federal do Ceará - UFC, Brasil. 3. Universidade de Fortaleza - Unifor, Brasil.

\begin{abstract}
Resumo
Introdução: A doença pulmonar obstrutiva crônica (DPOC) é classificada atualmente como importante causa de morbidade e mortalidade no mundo e está associada a importantes manifestações sistêmicas. $O$ body mass index airway obstruction, dyspnea and exercise capacity (BODE) é um índice prognóstico de mortalidade mais utilizado atualmente, pois avalia as manifestações respiratórias e sistêmicas da DPOC. Objetivo: Analisar, através do índice de BODE, os pacientes com DPOC severa participantes de um Programa de Reabilitação Pulmonar. Metodologia: Estudo documental, retrospectivo e quantitativo. Analisou-se o índice BODE e suas relações com outras variáveis fisiológicas. A amostra foi composta por 29 prontuários do Programa de Reabilitação Pulmonar, e investigaram-se os seguintes registros: idade, sexo, índice de massa corporal, VEF1, índice de dispneia modificada, Teste de caminhada de seis minutos, SF-36 e do índice BODE. Resultados: Dos pacientes analisados, 48,3\% (n = 14) eram do sexo feminino e 51,7\% (n = 15) masculino; idade $66 \pm 8,8$ anos; IMC 25,1 $\pm 4,4 \mathrm{~kg} / \mathrm{m}^{2}$. Verificaram-se as seguintes diferenças VEF1 0,98 $\pm 0,4$ e 0,95 $\pm 0,3$ (p = 0,26); MRC 2,40 $\pm 1,0$ e $1,76 \pm 0,6(p=0,005)$; BODE 4,0 $\pm 1,7$ e 3,29 $\pm 1,1(p=0,009)$; TC $6 \min 404,58 \pm 110,4$ e 439,96 \pm 97,3 ( $\mathrm{p}=0,02)$ antes e após o Programa de Reabilitação respectivamente. A correlação entre o índice BODE e o TC6' foi $r=-0,51$ e do índice BODE e VEF1 foi $r=-0,81$. Conclusão: Pode-se inferir que o Programa de Reabilitação Pulmonar modificou, de forma significativa, o índice de BODE influenciando a qualidade de vida dos pacientes.
\end{abstract}

Palavras-chave: Doença Pulmonar Obstrutiva Crônica. Índice de Gravidade de Doença. Reabilitação.

\begin{abstract}
Introduction: The Chronic Obtructive Pulmonary Disease (COPD) is a important cause of morbidity and mortality worldwide associated with systemic consequences. The body mass index airway obstruction, dyspnea and exercise capacity (BODE) index is a predictor of mortality most used today, because it assesses respiratory and systemic manifestations of COPD. Objective: To analyze the BODE index in patients with COPD severe participants of pulmonary rehabilitation program. Methodology: This is a documental, quantitative and retrospective study. This study was designed to investigate the association between BODE index and others physiological variables. The sample was composed by 29 patients in the Pulmonary Rehabilitation Program. The data were collected from records: age, sex, body mass index, FEV1, dyspnea index modified, six minutes walking test, SF-36 and BODE index. Results: Of the patients studied, $48.3 \%(\mathrm{n}=14)$ were female and $51.7 \%(\mathrm{n}=15)$ male; age $66 \pm 8.8$ years; BMI $25.1 \pm 4.4 \mathrm{~kg} / \mathrm{m}^{2}$. Changes were significantly improved in FEV1 $0.98 \pm 0.4$ and $0.95 \pm 0.3(\mathrm{p}=0.26)$; MMRC $2.40 \pm 1.0$ and $1.76 \pm 0.6(\mathrm{p}=0.005)$; BODE index $4.0 \pm 1.7$ and $3.29 \pm 1.1(\mathrm{p}=0.009)$ and $6 \mathrm{MWT} 404.58 \pm 110.4$ and $439.96 \pm 97.3(\mathrm{p}=0.02)$ before and after the rehabilitation program respectively. The correlation between the BODE index and the 6MWT was $r=-0.51$ and BODE index and FEV1 was $r=-0.81$. Conclusion: We may infer that Rehabilitation Program changed significantly the BODE index influencing the improvement quality of life the patients.
\end{abstract}

Key-words: Pulmonary Disease, Chronic Obstructive. Severity of Illness Index. Rehabilitation.

\section{INTRODUÇÃO}

A doença pulmonar obstrutiva crônica (DPOC) é classificada atualmente como importante causa de morbidade e mortalidade no mundo ${ }^{1}$. É difícil determinar a prevalência exata da DPOC, mas estudos epidemiológicos sugerem que afeta aproximadamente de 7 a 19\% da população, e está aumentando o número de casos em mulheres. Assumindo- se a menor prevalência, o total de casos se aproxima a 280 milhões de pessoas ${ }^{2-5}$.

No Brasil, a prevalência da DPOC pode atingir $12 \%$ da população com mais de 40 anos de idade e, nos últimos anos, ocupou da 4 a à 7a posição entre as principais causas de morte $^{6}$. 
ADPOC é uma enfermidade comum, geralmente progressiva e debilitante, caracterizada por limitação do fluxo aéreo, dispneia, alterações nutricionais, redução da capacidade ao exercício, da força muscular e da qualidade de vida. Esse conjunto de alterações associado à hiperinsuflação dinâmica, que impede o paciente de alcançar as demandas ventilatórias necessárias aos exercícios, favorece o surgimento da dispnéia ${ }^{1,7-9}$.

No paciente com DPOC, a dispneia ocorre aos mínimos esforços, em atividades da vida diária ou mesmo em repouso, portanto, ela é um dos fatores causadores do descondicionamento físico desses pacientes, o qual se deve, principalmente, à inatividade muscular ${ }^{1,10}$.

Essa doença não é totalmente reversível e está associada a uma resposta inflamatória do pulmão a partículas ou gases nocivos. Acontece após um ataque crônico e agressivo das pequenas vias aéreas e do parênquima pulmonar manifestando-se em proporções variáveis de indivíduo para indivíduo. Na maioria das vezes, o agressor é a fumaça de cigarro' . Porém, em muitos países, a poluição do ar resultante da queima de lenha, outros combustíveis e biomassas também têm sido identificados como fatores de risco para a DPOC ${ }^{11}$.

Essas manifestações, as quais incluem as alterações da qualidade de vida e da composição do corpo e as repercussões sobre a tolerância ao exercício, também estão associadas à frequência de exacerbações, às taxas de hospitalização e à sobrevida desses pacientes ${ }^{12}$.

Estudos recentes sugerem que outros parâmetros além do volume expiratório forçado no primeiro segundo $\left(V_{E F}\right)$, como índice de massa corpórea (IMC), grau de dispneia, distância percorrida avaliada pelo teste de caminhada de seis minutos $\left(\mathrm{TC}^{\prime}\right)$, qualidade de vida relacionada à saúde, pico de consumo de oxigênio $\left(\mathrm{VO}_{2}\right)$, relação de capacidade inspiratória/capacidade pulmonar total $(\mathrm{Cl} /$ $\mathrm{CPT}$ ), Índice BODE e $\theta$ uso de oxigênio a longo prazo, independentemente, são importantes fatores preditores de prognóstico em DPOC, e em alguns são, na verdade, melhores marcadores do que o $\mathrm{VEF}_{1}{ }^{7}$.

O Índice de BODE, do inglês body mass index, airway obstruction, dyspnea, and exercise capacity, é um índice prognóstico multidimensional para pacientes portadores de DPOC, baseado no IMC, no $\operatorname{VEF}_{1}$, na percepção de dispneia através da escala de dispneia Medical Research Council (MRC) e na capacidade de realizar exercícios por meio do desempenho do TC6 ${ }^{4,13}$.

Esse índice vem sendo mais utilizado atualmente para avaliar pacientes com DPOC porque não avalia o $\mathrm{VEF}_{1}$ isoladamente para não mascarar os resultados; analisa as manifestações respiratórias e sistêmicas da DPOC e pode caracterizar e predizer melhor os desfechos nesses pacientes ${ }^{8}$.

O paciente portador de DPOC diminui sua atividade física global devido à piora progressiva da função pulmonar.
O progressivo descondicionamento físico associado à inatividade dá início a um círculo vicioso, em que a piora da dispneia se associa a esforços físicos cada vez menores, com grave comprometimento da qualidade de vida ${ }^{14}$.

A estratégia utilizada pelo Programa de Reabilitação Pulmonar é integrar-se ao manejo clínico e à manutenção da estabilidade clínica dos portadores de DPOC, principalmente nos pacientes que, mesmo com tratamento clínico otimizado, continuam sintomáticos e com diminuição de sua função física e social ${ }^{15}$.

A reabilitação pulmonar não beneficia o paciente no seu quadro de obstrução ao fluxo aéreo, mas auxilia-o, diminuindo as deficiências e as disfunções sistêmicas consequentes dos processos secundários da doença pulmonar, como as disfunções musculares periféricas e respiratórias, as anormalidades nutricionais, as deficiências cardiovasculares, os distúrbios esqueléticos, sensoriais e psicossociais ${ }^{16}$.

Nesse contexto, idealizamos um estudo pioneiro no Estado do Ceará com o intuito de avaliar a utilização do índice de Bode em um Programa de Reabilitação Pulmonar, já que este índice está se destacando atualmente por sua capacidade de caracterizar, com maior fidedignidade, o prognóstico do paciente com DPOC. Além disso, correlacionar o índice de BODE com o volume expiratório forçado no primeiro segundo, o índice de dispneia, o teste de caminhada de 6 minutos e a qualidade de vida.

Esse estudo objetiva analisar o índice BODE e suas relações com outras variáveis fisiológicas em pacientes com DPOC antes e após participarem do Programa de Reabilitação Pulmonar.

\section{METODOLOGIA}

Estudo documental, retrospectivo e quantitativo, realizado por meio da análise de prontuários de pacientes com DPOC que participaram do Programa de Reabilitação Pulmonar na Unidade de Reabilitação Cardiorrespiratória do Hospital de Messejana Dr. Carlos Alberto Studart Gomes, em Fortaleza, Ceará.

O estudo foi realizado após aprovação do Comitê de Ética e Pesquisa do Hospital de Messejana Dr. Carlos Alberto Studart Gomes e seguiu respeitando as normas da Resolução n. 196/96 do Conselho Nacional de Saúde pesquisa envolvendo seres humanos ${ }^{17}$.

Foram utilizados dados dos prontuários de pacientes do PRP que participaram no período de janeiro a dezembro de 2009. Os critérios de inclusão foram: prontuários de pacientes que tenham participado regularmente do PRP no período mínimo de três meses, estabelecido pelo protocolo do serviço, independentes de religião, sexo, cor, idade e raça e que tenham o diagnóstico comprovado de DPOC através da espirometria, exame de função pulmonar considerado padrão ouro para esse diagnóstico. 
Foram excluídos da pesquisa os prontuários de pacientes que interromperam a participação no PRP por qualquer causa, os pacientes que tinham Insuficiência Cardíaca Congestiva associada e os que desistiram ou abandonaram o tratamento.

A avaliação da obstrução das vias aéreas tem um papel importante no diagnóstico e estadiamento da DPOC, sendo analisado através do $\mathrm{VEF}_{1}$.

A relação $V F_{1} / C V F$ (Índice de Tiffeneau) menor que 0,7 após o uso de broncodilatador é critério espirométrico necessário para estabelecer o diagnóstico da DPOC. Os critérios espirométricos para estadiamento da DPOC conforme o Global Initiative for Chronic Obstructive Lung Disease (GOLD) são: DPOC leve com $\mathrm{VEF}_{1} \geq 80 \%$ do previsto e VEF $_{1} /$ CVF < 0,7; DPOC moderado com $50 \% \leq \mathrm{VEF}_{1}<80 \%$

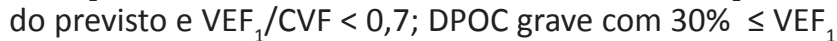
$<50 \%$ do previsto e $\mathrm{VEF}_{1} / \mathrm{CVF}<0,7$; DPOC muito grave com $\mathrm{VEF}_{1}<30 \%$ do previsto ou $\mathrm{VEF}_{1}<50 \%$ do previsto associado à insuficiência respiratória crônica e $\operatorname{VEF}_{1} / C V F<0,7^{11}$.

Os dados foram registrados em formulário próprio para registro dos parâmetros que foram analisados no estudo (IMC, $\mathrm{VEF}_{1}, \mathrm{MRC}$, Teste de Caminhada de 6 minutos, SF-36 e BODE).

- $\mathrm{IMC}=$ medido pelo peso/altura ${ }^{2}$, é uma medida do grau de obesidade de uma pessoa; possibilita classificar se o voluntário está acima ou abaixo dos parâmetros ideais de peso para sua estatura;

- VEF1 = corresponde à quantidade máxima de ar expirado durante o primeiro segundo da manobra expiratória forçada; é importante parâmetro para observação do grau de obstrução pulmonar ${ }^{7,12}$;

- Medical Research Council (MRC) = Índice de dispneia segundo o MRC, com o intuito de obervar a gravidade da doença: 0 - falta de ar em exercícios intensos; 1 - falta de ar surge quando o paciente apressa o passo no plano ou sobe escadas ou ladeiras; 2 - dispneia no próprio passo no plano ou dificuldade para acompanhar o passo de outra pessoa da mesma idade; 3 - dispneia no plano em menos de 100 metros ou após alguns minutos de caminhada e 4 - dispneia para sair de casa ou pra se vestir ${ }^{18}$;

- TC6 $^{\prime}$ = distância percorrida pelo paciente em seis minutos, a qual reflete a capacidade funcional dos pacientes com DPOC, sendo um fator de predição da mortalidade em pacientes com doença grave;

- SF-36 = é um questionário genérico multidimensional, formado por 36 itens, englobados em oito domínios. A qualidade de vida é classificada segundo os escores que variam de 0 (mínimo) a 100 (máximo), sendo que os maiores escores indicam melhor qualidade de vida. O questionário é dividido em dois componentes: físicos (CFS) e mentais (CMS). O componente físico é formado pelos domínios capacidade funcional (com dez itens), estado geral da saúde (com cinco itens), dor (com dois itens) e aspectos físicos (com quatro itens). O componente mental é constituído pelos domínios saúde mental (com cinco itens), vitalidade (com quatro itens), aspectos sociais (com dois itens) e aspectos emocionais (com três itens). Este representa o conceito de saúde mais frequentemente utilizado nas pesquisas em saúde ${ }^{19-20}$.

- $\mathrm{BODE}=$ os parâmetros são avaliados e pontuados de 0 a 3 , e os resultados são somados em um no total de 0 a 10. Com esse índice, conclui-se que quanto maior a pontuação, pior o prognóstico e maior o índice de mortalidade $\mathrm{e}^{2,4,13}$.

A estatística descritiva e inferencial foi realizada por intermédio da ferramenta estatística do software Microsoft Office Excel 2007C. Utilizou-se o teste t de Student, e consideram-se significativos os valores de $p<0,05$ (5\%). As correlações entre as variáveis contínuas foram analisadas pelo Teste de Pearson.

\section{RESULTADOS}

A amostra foi composta por 29 indivíduos portadores de DPOC participantes do Programa de Reabilitação Pulmonar (PRP), sendo $48,3 \%(n=14)$ do sexo feminino e $51,7 \%$ ( $n=$ 15) do sexo masculino. A média da idade dos indivíduos foi $66( \pm 8,8)$ anos, variando de 47 a 79 anos. Em relação ao índice de massa corporal (IMC), a média foi de 25,1 $( \pm 4,4)$ $\mathrm{kg} / \mathrm{m}^{2}$, sendo classificados como sobrepeso. De acordo com a classificação GOLD, 3,6\% ( $n=1)$ tinha DPOC Leve, $28,6 \%$ ( $n=8)$ Moderado, 50\% ( $n=14)$ Grave e 17,8\% ( $n=5)$ muito Grave. Toda a amostra foi composta por ex-tabagistas.

Dos indivíduos que participaram do PRP 93,1\% ( $n=26)$ fazia uso de oxigênio durante a realização do exercício físico. Em relação ao tempo de participação no PRP, 12 pacientes participaram por 3 a 5 meses, e 17 participaram por 6 a 8 meses. O tempo médio de permanência desses pacientes no programa foi de 5,9 $( \pm 1,6)$ meses.

A Tabela 1 mostra os valores médios das variáveis que compõem o Índice BODE. $\mathrm{Na}$ análise das variáveis isoladamente, $\mathrm{o} \mathrm{VEF}_{1}$ foi o único que não apresentou diferença estatística significativa ( $p=0,26$ ) após o PRP, porém não afetando a melhora do Índice de BODE.

Tabela 1. Distribuição das variáveis do estudo antes e após a participação dos pacientes no Programa de Reabilitação Pulmonar.

\begin{tabular}{llll}
\hline Variáveis & Antes & Após & $\boldsymbol{p}$ \\
\hline VEF $_{1}$ & $0,98 \pm 0,4$ & $0,95 \pm 0,3$ & 0,26 \\
MRC & $2,4 \pm 1,0$ & $1,76 \pm 0,6$ & $0,005^{*}$ \\
BODE & $4,0 \pm 1,7$ & $3,29 \pm 1,1$ & $0,009^{*}$ \\
SF-36 & & & \\
CFS & $36,31 \pm 10,5$ & $39,48 \pm 9,5$ & $0,02^{*}$ \\
CMS & $45,84 \pm 11,7$ & $52,34 \pm 11,9$ & $0,001^{*}$ \\
TC 6min & $404,58 \pm 110,4$ & $439,96 \pm 97,3$ & $0,02^{*}$ \\
\hline
\end{tabular}

Fonte: HM/Fortaleza, Ceará, 2010. ${ }^{*} p<0,05$; teste t de Student pareado. 
As mesmas variáveis foram analisadas em função do sexo. O Índice BODE não alterou-se nas mulheres participantes do Programa de Reabilitação Pulmonar $(p=0,11)$ (Tabela 2). Porém, verificou-se significância estatística na distância percorrida do TC6' $(p=0,02)$ bem como no componente mental (CMS) do SF-36 ( $p=0,04)$.

Tabela 2. Distribuição das variáveis do estudo antes e após a participação dos pacientes no Programa de Reabilitação Pulmonar para o sexo feminino.

\begin{tabular}{|c|c|c|c|}
\hline \multicolumn{4}{|l|}{ Mulheres } \\
\hline Variáveis & Antes & Após & $\mathrm{p}$ \\
\hline VEF1 & $0,73 \pm 0,2$ & $0,75 \pm 0,2$ & 0,4 \\
\hline $\mathrm{MRC}$ & $2,30 \pm 0,8$ & $1,84 \pm 0,5$ & 0,05 \\
\hline BODE & $4,07 \pm 1,5$ & $3,53 \pm 0,9$ & 0,11 \\
\hline \multicolumn{4}{|l|}{ SF-36 } \\
\hline CFS & $37,44 \pm 12,9$ & $40,00 \pm 8,6$ & 0,15 \\
\hline CMS & $43,26 \pm 13,5$ & $49,18 \pm 12,5$ & $0,04^{*}$ \\
\hline TC 6min & $399,78 \pm 97,2$ & $432,5 \pm 90,5$ & $0,02 *$ \\
\hline \multicolumn{4}{|c|}{$\begin{array}{l}\text { Fonte: } \mathrm{HM} / \text { Fortaleza, Ceará, } 2010 .{ }^{*} \mathrm{p}<0,05 \text {; teste t de Student } \\
\text { pareado. }\end{array}$} \\
\hline \multicolumn{4}{|c|}{$\begin{array}{l}\text { Quando analisado o sexo masculino }(n=15) \text { (Tabela } 3) \text {, } \\
\text { observou-se diminuição significativa }(p=0,01) \text { do índice } \\
\text { de BODE, provavelmente influenciado pela diminuição } \\
\text { significativa }(p=0,02) \text { do índice de dispneia (MRC). Os } \\
\text { valores referentes ao componente mental ( } p=0,003) \\
\text { e físico ( } p=0,03) \text { do SF-36 apresentaram aumentos } \\
\text { significativos, ocasionando uma melhora no prognóstico } \\
\text { desses pacientes. De acordo com a classificação GOLD, } \\
\text { os participantes do sexo masculino apresentavam menor }\end{array}$} \\
\hline
\end{tabular}

gravidade, apenas sete pacientes foram classificados como grave e muito grave ao passo que havia 12 mulheres com esta classificação.

Tabela 3. Distribuição das variáveis do estudo antes e após a participação dos pacientes no Programa de Reabilitação Pulmonar para o sexo masculino.

\begin{tabular}{|c|c|c|c|}
\hline \multicolumn{4}{|l|}{ Homens } \\
\hline Variáveis & Antes & Após & $\mathrm{p}$ \\
\hline VEF1 & $1,23 \pm 0,5$ & $1,15 \pm 0,41$ & 0,18 \\
\hline $\mathrm{MRC}$ & $2,5 \pm 1,3$ & $1,6 \pm 0,7$ & $0,02 *$ \\
\hline BODE & $3,90 \pm 2,1$ & $3,0 \pm 1,4$ & $0,01^{*}$ \\
\hline \multicolumn{4}{|l|}{$S F-36$} \\
\hline CFS & $35,25 \pm 8,0$ & $38,99 \pm 10,6$ & $0,03^{*}$ \\
\hline CMS & $48,25 \pm 9,5$ & $55,29 \pm 10,9$ & $0,003^{*}$ \\
\hline TC $6 \mathrm{~min}$ & $\begin{array}{l}409,06 \pm \\
124,7\end{array}$ & $446,9 \pm 106,0$ & 0,1 \\
\hline
\end{tabular}

Ao avaliar a amostra pelo período de participação no Programa de Reabilitação Pulmonar, observou-se que $41,3 \%(n=12)$ dos pacientes ficaram por 3 a 5 meses e $58,7 \%(n=17)$ por 6 a 8 meses. No grupo que participou por 3 a 5 meses, os participantes apresentaram resultados estatísticos significativos para o Índice de BODE $(p=0,008)$ e o SF-36, componente físico $(p=0,003)$ e mental $(p=0,009)$. No grupo que permaneceu por 6 a 8 meses, os resultados foram significativos para escala de $\operatorname{MRC}(p=0,01), C M S(p=$ $0,02)$ e TC6' $(p=0,03)$ (Tabela 4).

Tabela 4. Distribuição das variáveis do estudo antes e após a participação dos pacientes no Programa de Reabilitação Pulmonar em relação ao tempo de participação.

\begin{tabular}{|c|c|c|c|c|c|c|}
\hline \multicolumn{7}{|c|}{ Reabilitação Pulmonar } \\
\hline & 3 a 5 meses & & & 6 a 8 meses & & \\
\hline Variáveis & Antes & Após & $\mathrm{p}$ & Antes & Após & $\mathbf{p}$ \\
\hline VEF1 & $1,16 \pm 0,5$ & $1,42 \pm 0,4$ & 0,41 & $0,86 \pm 0,4$ & $0,83 \pm 0,3$ & 0,23 \\
\hline $\mathrm{MRC}$ & $2,4 \pm 1,3$ & $1,8 \pm 0,7$ & 0,09 & $2,4 \pm 0,9$ & $1,7 \pm 0,5$ & $0,01 *$ \\
\hline BODE & $4,33 \pm 2,0$ & $3,3 \pm 1,4$ & $0,008 *$ & $3,66 \pm 1,6$ & $3,2 \pm 1,1$ & 0,12 \\
\hline \multicolumn{7}{|l|}{ SF-36 } \\
\hline CFS & $36,69 \pm 8,9$ & $42,55 \pm 9,0$ & $0,003^{*}$ & $36,04 \pm 11,8$ & $37,13 \pm 9,3$ & 0,3 \\
\hline CMS & $48,35 \pm 8,8$ & $55,59 \pm 10,8$ & $0,009 *$ & $44,07 \pm 13,3$ & $50,05 \pm 12,5$ & $0,02 *$ \\
\hline TC 6 min & $414 \pm 139,4$ & $463 \pm 113,7$ & 0,1 & $397,3 \pm 88,5$ & $423,7 \pm 83,7$ & $0,03 *$ \\
\hline
\end{tabular}

Fonte: HM/Fortaleza, Ceará, 2010. $* \mathrm{p}<0,05$; teste t de Student pareado. 
Investigou-se também, neste estudo, a correlação do Índice de BODE com a capacidade funcional traduzida pelo teste de caminhada de seis minutos por meio da correlação de Pearson. Os resultados da análise do Índice de BODE e TC6' após a RP foi de $r=-0,51$, mostrando que, em $51 \%$ dos casos, ocorreu uma tendência à diminuição do Índice BODE e ao aumento da distância caminhada, possivelmente indicando que esses pacientes apresentaram uma melhora no quadro clínico e prognóstico (Gráfico 1).

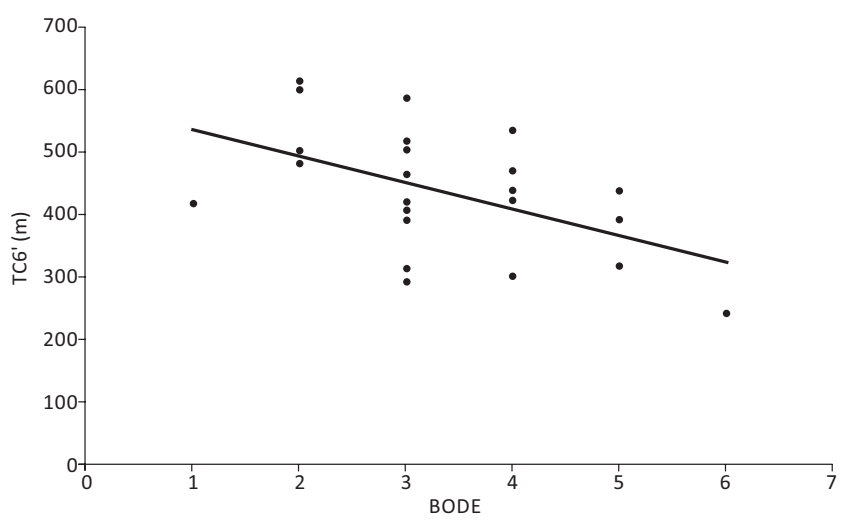

Gráfico 1. Correlação entre o índice BODE e o Teste de Caminhada de 6 minutos.

O Índice de BODE e o $\mathrm{VEF}_{1}$ apresentaram uma relação fortemente negativa $(r=-0,81)$, como já esperado, pois o Índice de BODE, por ser uma medida multifatorial, tem como um de seus componentes o $\mathrm{VEF}_{1}$, e este não se altera em resultado ao treinamento proposto no PRP (Gráfico 2).

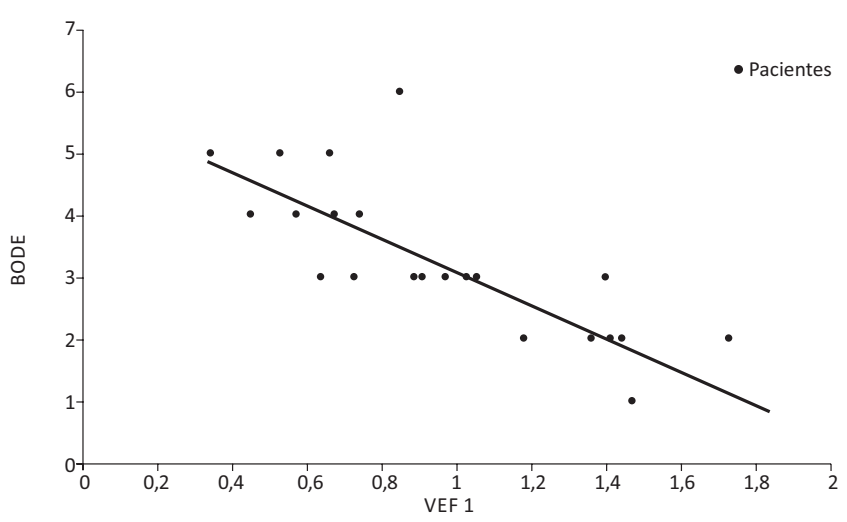

Gráfico 2. Correlação entre VEF1 e o índice de BODE.

\section{DISCUSSÃO}

Na DPOC, a perda da capacidade elástica pulmonar e a limitação expiratória progressiva promovem aprisionamento aéreo, com aumento da capacidade residual funcional (CRF) e diminuição da capacidade inspiratória $(\mathrm{Cl})$. A hiperinsuflação estática e seu aumento durante o exercício (hiperinsuflação dinâmica) se associam à limitação ao exercício em portadores de DPOC. E a redução da hiperinsuflação, expressa pelo aumento da $\mathrm{Cl}$ pós-broncodilatador, correlaciona-se com melhor tolerância ao exercício e à redução da dispneia?

Outros fatores contribuem para a limitação desses pacientes a atividade física como anormalidades de troca gasosa, hipertensão pulmonar, doenças relacionados ao tabagismo como hipertensão e doença cardíaca. O teste de caminhada de seis minutos (TC6') reflete todos esses fatores, incluindo os efeitos sistêmicos associados à doença e à limitação ventilatória ${ }^{7,21}$.

No presente estudo dos pacientes que participaram do PRP, o VEF ${ }_{1}$ foi o único parâmetro que não apresentou diferença significativa após a reabilitação; sendo assim, Freitas et al. ${ }^{7}$ afirmam que, embora, tradicionalmente, a gravidade da DPOC seja definida pelo grau de obstrução avaliado pelo $\mathrm{VEF}_{1}$ após o uso de broncodilatador, este não se correlaciona com a dispneia, que é o sintoma mais importante do portador de DPOC, também não é bom marcador de sobrevida, frequência de internações e capacidade funcional, principalmente no paciente mais grave. O paciente portador de DPOC tem manifestações sistêmicas que não são representadas pelo $\mathrm{VEF}_{1}{ }^{5}$.

Simon et al. ${ }^{1}$ acrescentam que pacientes que possuem grau de obstrução pulmonar elevado podem limitar as atividades físicas, porém a atividade física não exerce influência na variável $\mathrm{VEF}_{1}$ desses indivíduos.

Comprovando nossos achados, Bott e Singh ${ }^{22}$ afirmam que entre os objetivos do PRP estão: maximizar a independência funcional do indivíduo em suas atividades de vida diária, minimizar a dependência de outras pessoas ou órgãos secundários, avaliar e iniciar, quando necessário, o treinamento físico para melhorar a capacidade física do paciente, proporcionar sessões educativas a pacientes, familiares e outras pessoas envolvidas em relação ao processo da doença, medicação e técnicas terapêuticas.

Quando comparamos as variáveis em relação ao sexo, o Índice de BODE não foi diferente significativamente nas mulheres participantes do PRP, entretanto, observamos significância estatística no TC6' e no CMS do SF-36, já em relação ao sexo masculino, observamos diferenças significativas no Índice de BODE, no MRC e na qualidade de vida após o PRP, porém apenas sete homens eram pacientes graves ou muito graves ao passo que havia 12 mulheres com essa classificação, segundo o GOLD.

Corroborando com nossos resultados, Atalaia ${ }^{23}$ destaca que o treino aeróbio de baixa intensidade realizado em esteira ergométrica, bicicleta ou a simples marcha leva a melhorias significativas na tolerância ao exercício mesmo sem ganhos fisiológicos apreciáveis. Em relação ao sexo feminino, o estilo de vida, os valores culturais que impõem à mulher grande responsabilidade doméstica além da atividade profissional justificam o benefício do PRP no componente mental. 
Dreyse et al. ${ }^{3}$ compararam esses parâmetros entre os gêneros e não observaram diferenças estatísticas na severidade da doença, indo de encontro aos nossos resultados, entretanto, no grupo das mulheres, encontraram o mesmo grau de severidade com um menor tempo de exposição ao fumo. Os autores explicam que isso pode ocorrer pelas características anatômicas femininas que possuem um pulmão e vias aéreas inferiores menores.

O esforço exigido no TC6' reflete o esforço realizado nas atividades da vida diária e a baixa distância percorrida nesse teste reproduz suas limitações ${ }^{5}$. Verificou-se, neste estudo, que, tanto na análise geral quanto na análise por grupo, o sexo feminino apresentou melhora significativa da distância percorrida no TC6'.

Zanchet et al. ${ }^{24}$ relatam que a distância percorrida no teste de caminhada de seis minutos é menor em portadores de DPOC do que em indivíduos saudáveis, e um aumento de $54 \mathrm{~m}$ na distância percorrida é clinicamente significativo. Em nosso estudo, embora a diferença tenha sido menor do que $54 \mathrm{~m}$, houve melhora clínica confirmada pelo SF-36 e Índice de BODE. A distância percorrida no teste de caminhada teve um aumento de $35 \mathrm{~m}$ e mostrou significância estatística $(p=0,02)$ em relação ao inicial.

Em relação ao tempo de participação no PRP, observamos que, de três a cinco meses, ocorreu uma melhora significativa para o Índice de BODE e o SF-36 e, no grupo seis a oito meses, a melhora significativa se deu no MRC, CMS e TC6', corroborando nossos resultados. Pitta et al. ${ }^{25}$, afirmam que programas de reabilitação pulmonar de longa duração com orientação no tocante às mudança nos hábitos da vida diária levam à melhora do tempo de caminhadas na vida diária e, consequentemente, ocorrem melhoras significativas da dispneia e do teste de caminhada em seis minutos.

Os transtornos psicológicos estão-se tornando cada vez mais reconhecidos como importantes comorbidades em pacientes com DPOC. E, apesar de os mecanismos dessas associações serem pouco conhecidos, elas são suscetíveis a intervenções terapêuticas, influenciando em fatores como depressão e ansiedade, que são fatores de risco para rehospitalizações e pior sobrevida ${ }^{26-27}$. Nesse estudo, pode-se verificar a melhora dos pacientes de acordo com a análise do questionário de qualidade de vida SF-36 na análise geral da amostra, bem como para o sexo feminino e masculino.

A atividade física está ligada a um melhor prognóstico para indivíduos com DPOC. As diretrizes publicadas pelo American College of Sports Medicine (ACSM) recomendam no mínimo 30 minutos de atividade física de intensidade moderada (por ex., caminhada) para a manutenção ou o desenvolvimento da aptidão física, independentemente da idade $^{28}$

Os pacientes que não atendam a esses padrões mínimos são considerados insuficientemente ativos e apresentam um maior risco de morbimortalidade. A atividade física na vida cotidiana de pacientes com DPOC é muito limitada e se deteriora ainda mais com o tempo devido a fatores como as crises agudas, fazendo que aumente ainda mais o grau de mortalidade dessa população ${ }^{8}$. Os pacientes gravemente comprometidos pela doença apresentaram um aumento significativo do índice de BODE para cada dia de inatividade física ${ }^{1}$.

Apesar de o PRP não proporcionar nítida melhoria funcional respiratória, beneficia outras áreas de interesse do doente, como a dispneia, a capacidade de realizar exercícios, o estado geral de saúde, as atividades da vida diária e os sintomas relacionados com a saúde e a qualidade de vida $^{23,29}$.

Vasconcelos et al. ${ }^{14}$ confirmam nossos resultados, quando destacam que o programa de reabilitação pulmonar promove um aumento da independência funcional, melhora a capacidade de realizar exercícios e atividades rotineiras, influenciando diretamente a qualidade de vida dos seus participantes. Os exercícios aeróbios se mostram importantes na melhora da capacidade motora, na diminuição da fadiga muscular e do descondicionamento físico, reduzindo, assim, o sedentarismo.

Godoy et al. ${ }^{27}$ reavaliaram pacientes que participaram de um programa de reabilitação pulmonar após dois anos de conclusão e sem terem contato com a equipe multidisciplinar de reabilitação e verificaram que os benefícios obtidos a curto prazo relacionados à redução dos níveis de sintomas de ansiedade e depressão, à melhora do índice de qualidade de vida e ao aumento na distância caminha no TC6' se mantiveram a longo prazo.

Entre as limitações do presente estudo, destaca-se a avaliação dos pacientes de um único Programa de Reabilitação Pulmonar; entretanto, destacamos que o programa escolhido é o pioneiro e um dos mais conceituados no Estado do Ceará; é também, o que apresenta o maior número de pacientes em atividade. Desse modo, propomos que novos estudos utilizando uma maior amostra e maior tempo de observação após a reabilitação, com o objetivo de observar como os pacientes se comportam com o passar do tempo, além de estudos multicêntricos, envolvendo outros Programas de Reabilitação Pulmonar, podem ser realizados.

\section{CONCLUSÃO}

Evidenciamos que o Programa de Reabilitação Pulmonar modificou, de forma significativa, o grau de dispneia, o Índice de BODE, os componentes físico e mental do SF36 e a distância percorrida pelo teste de caminhada de seis minutos. Entretanto, não observamos alterações significativas no $V F_{1}$, possivelmente por os exercícios utilizados no Programa de Reabilitação Pulmonar não proporcionarem alterações no grau de obstrução pulmonar e sim na capacidade de tolerar exercícios, independência funcional e qualidade de vida. 
Os resultados no sexo feminino mostraram diferença estatística apenas no TC6' e componente mental do SF-36, confirmando o pensamento de que a gravidade do DPOC implica pior quadro clínico e prognóstico.

O Programa de Reabilitação Pulmonar proporcionou aos pacientes que participaram pelo período de três a cinco meses alterações no Índice de BODE e componentes físico e mental do SF-36 e, no grupo que participou de seis a oito meses, a melhora ocorreu no índice de dispneia (MRC), no componente mental do SF-36 e no teste de caminhada de seis minutos, remetendo que, ao longo do tempo, as orientações direcionadas às mudanças nos hábitos de vida proporcionaram aumento do tempo de caminhada e diminuição da dispneia.

Ressalta-se que o treino físico e a manutenção dele nos indivíduos portadores de doença pulmonar obstrutiva crônica com diagnóstico precoce pode ocasionar um retardo na progressão natural da doença e na diminuição do risco de morbimortalidade, tornando-os suficientemente ativos.

\section{REFERÊNCIAS}

1. Simon KM, Hass AP, Zimmermman JL, Carpes MF. Índice prognóstico de mortalidade BODE e atividade física em doentes pulmonares obstrutivos crônicos. Rev Bras Med Esporte 2009; 15(1):19-23. doi: http://dx.doi.org/10.1590/S1517-86922009000100004.

2. Celli BR. Update on the Management of COPD. Chest 2008; 133(6):1451-62. doi: http://dx.doi.org/10.1378/chest.07-2061. PubMed PMID:18574288.

3. Dreyse JD, Lisboa CB, Pinto CR, Saldías FP, Díaz OP. Características clínicas y funcionales según gênero de pacientes com enfermedad pulmonar obstructiva crônica. Rev. Chil. Enferm. Respir 2008; 24(2):95100. doi: http://dx.doi.org/10.4067/S0717-73482008000200002.

4. Baldisserotto SV. Densitovolumetria em doença pulmonar obstrutiva crônica: correlação do volume de zonas hipoatenuadas com valores de referência da normalidade e o Índice "BODE" [tese]. Porto Alegre: Universidade Federal do Rio Grande do Sul; 2005.

5. Simon KM. Atividade de vida diária e índice de mortalidade "BODE" em indivíduos portadores de doença obstrutiva crônica [dissertação]. Uberlândia: Centro Universitário do Triângulo - UNITRI; 2006.

6. Roceto LS, Takara LS, Machado L, Zambon L, Saad IAB. Effectiveness of pulmonary rehabilitation once a week for patients with obstructive pulmonary disease. Rev Bras Fisioter 2007; 11(6):475-80.

7. Freitas CG, Pereira CA, Viegas CA. Inspiratory capacity, exercise limitation, markers of severity, and prognostic factors in chronic obstructive pulmonary disease. J Bras Pneumol 2007; 33(4):38996. doi: http://dx.doi.org/10.1590/S1806-37132007000400007. PubMed PMID:17982530

8. Pitta F, Troosters T, Probst VS, Lucas S, Decramer M, Gosselink R. Potential consequences for stable chronic obstructive pulmonary disease patients who do not get the recommended minimum daily amount of physical activity. J Bras Pneumol 2006; 32(4):301-08. doi: http://dx.doi. org/10.1590/S1806-37132006001100008. PubMed PMID: 17268729.

9. Campos LEM, Figueiredo CFTA. Síndromes pulmonares e pleurais. In: Lopéz M, Medeiros JL. Semiologia Médica - As bases do diagnóstico clínico. 5. ed. Rio de Janeiro: Revinter; 2004. p. 580-87.

10. Brunetto AF, Pitta FO, Probst VS, Paulin E, Yamaguti WPS, Ferreira LF. Influência da saturação de $\mathrm{O} 2$ na velocidade do teste de caminhada percorrida em 6 minutos, em pacientes com DPOC grave. Rev Bras Fisioter 2003; 7(2):123-29.

11. Global Initiative for Chronic Obstructive Lung Disease - GOLD. Estratégia global para o diagnóstico, condução e prevenção da doença pulmonar obstrutiva crônica [Internet]. Oregon: MCR Vision Inc.; 2006 [acesso em 15 Set 2010]. 112 p. Disponível em: http:// www.golddpoc.com.br/arquivos/GOLD-Report-Portugues.pdf.
12. Godoy I. Avaliando a gravidade e o prognóstico da doença pulmonar obstrutiva crônica: a medida do VEF1 ainda é suficiente? J Bras Pneumol 2007; 33 (4):xxiii-xiv. doi: http://dx.doi.org/10.1590/S180637132007000400002. PubMed PMID: 17982525. Editorial.

13. Celli BR, Cote CG, Marin JM, Casanova C, Montes OM, Mendez RA, Pinto PV, Cabral HJ. The body-mass index, airflow obstruction, dyspnea, and exercise capacity index in chronic obstructive pulmonary disease. N Engl J Med 2004; 350(10):1005-12. doi: http:// dx.doi.org/10.1056/NEJMoa021322. PubMed PMID: 14999112.

14. Vasconcelos TB, Josino JB, Bastos VPD, Nogueira ANC, Viana SMNR, Macena RHM. Exercícios aeróbios na reabilitação pulmonar: revisão de literatura. Anais do IV Seminário Internacional de Promoção da Saúde, Universidade de Fortaleza, p. 140, 2012.

15. Rodrigues SL, Viegas CAA, Lima T. Efetividade da reabilitação pulmonar como tratamento coadjuvante da doença pulmonar obstrutiva crônica. J. Pneumologia 2002; 28(2):65-70. doi: http:// dx.doi.org/10.1590/S0102-35862002000200002.

16. Galvez DS, Malaguti C, Battagim AM, Nogueira A, Velloso M. Evaluation of learning in patients with chronic obstructive pulmonary disease during a pulmonary Rehabilitation program. Rev Bras Fisioter 2007; 11(4):273-77.

17. Conselho Nacional de Saúde (BR). Resolução n . 196/96, de 10 de outubro de 1996. Aprovam diretrizes e normas regulamentadoras de pesquisa envolvendo seres humanos. Diário Oficial da União, Seção 1, p. 21086; 1996.

18. Smith J, Albert P, Bertella E, Lester J, Jack S, Calverley P. Qualitative aspects of breathlessness in health and disease. Thorax 2009; 64(8):713-8. doi: http://dx.doi.org/10.1136/thx.2008.104869. PubMed PMID:19386585.

19. Ciconelli RM, Ferraz MB, Santos W, Meinao I, Quaresma MR. Traduçao para a língua portuguesa e validaçao do questionário genérico de avaliaçao de qualidade de vida SF-36 (Brasil SF-36). Rev Bras Reumatol 1999; 39(3):143-150.

20. Vasconcelos TB, Barbosa EA, Olivério NCP, Enéas RA, Xavier EP. Avaliação da qualidade de vida de pacientes amputados transtibiais unilaterais antes e após a protetização. Fisioter Brasil 2011; 12(4):291-97.

21. Johnson JE. Which exercise test should be used for patients with symptomatic COPD? Chest. 2004; 126(3):668-70. doi: http://dx.doi. org/10.1378/chest.126.3.668. PubMed PMID:15364738.

22. Bott J, Singh SJ. Reabilitação Pulmonar. In: Pryor JA, Webber BA. Fisioterapia para problemas respiratórios e cardíacos. 2. ed. Rio de Janeiro: Guanabara Koogan; 2002. p. 264-75. 
23. Atalaia H. Intervenção da fisioterapia em utentes com doença pulmonar obstrutiva crônica. Rev Ess fisionline. 2007; 3(2):28-40.

24. Zanchet RC, Viegas CAA, Lima T. Efficacy of pulmonary rehabilitation: exercise capacity, respiratory muscle strength and quality of life in patients with chronic obstructive pulmonary disease. J Bras Pneumol. 2005; 31(2):118-24. doi: http://dx.doi.org/10.1590/S180637132005000200006 .

25. Pitta F, Troosters T, Probst VS, Langer D, Decramer M, Gosselink R. Are patients with COPD more active after pulmonary rehabilitation?. Chest 2008; 134(2):273-80. doi: http://dx.doi.org/10.1378/ chest.07-2655. PubMed PMID:18403667.

26. Funk GC, Kirchheiner K, Burghuber OC, Hartl S. BODE índex versus GOLD classification for explaining anxious and depressive symptoms in patients with COPD - a cross-sectional study. Respiratory Res 2009; 10(1):1-8. doi: http://dx.doi.org/10.1186/1465-9921-10-1. PubMed PMID: 19134192; PubMed Central PMCID: PMC2626579.
27. Godoy RF, Teixeira PJZ, Becker-Junior B, Michelli M, Godoy DV. Long-term repercussions of a pulmonary rehabilitation program on the indices of anxiety, depression, quality of life and physical performance in patients with COPD. J Bras Pneumol 2009; 35(2):12936. doi: http://dx.doi.org/10.1590/S1806-37132009000200005. PubMed PMID: 19287915.

28. Pate RR, Pratt M, Blair SN, Haskell WL, Macera CA, Bouchard C et al. Physical activity and public health. A recommendation from the Centers for Disease Control and Prevention and the American College of Sports Medicine. JAMA. 1995;273(5):402-7. doi: http://dx.doi. org/10.1001/jama.1995.03520290054029. PubMed PMID: 7823386.

29. Pamplona P, Morais L. Treino de exercício na doença pulmonar crónica. Rev Port Pneumol. 2007; 13(1):101-28. 